\title{
Enzyme Multilayer-Modified Biosensors. Use of Streptavidin and Deglycosylated Avidin for Constructing Glucose Oxidase and Lactate Oxidase Multilayers
}

\author{
Jun-ichi ANZAI ${ }^{\dagger}$, Yuka KobaYASHI and Hiroki TaKeSHITA \\ Faculty of Pharmaceutical Sciences, Tohoku University, Aramaki, Aoba-ku, Sendai 980-77, Japan
}

Keywords Enzyme multilayer, biosensor, streptavidin, deglycosylated avidin, biotin-labeled enzyme

We have reported that an enzyme can be built in a layer-by-layer structure of protein multilayers on an electrode surface, by depositing avidin and biotin-labeled enzyme alternately (Fig. 1). ${ }^{1-3}$ The enzyme multilayermodified electrodes thus prepared function as enzyme biosensors and their performance characteristics can be controlled by regulating the number of the enzyme layers on the electrode. Based on this technique, glucose ${ }^{1}$, lactate $^{2}$, and alcohol sensors ${ }^{3}$ have been prepared so far. The formation of the enzyme multilayers relies on a noncovalent and strong binding between avidin and biotin residues in the biotin-labeled enzymes (binding constant: ca. $\left.10^{15} \mathrm{M}^{-1}\right) .^{4}$ It is anticipated that the enzyme multilayers can be constructed also using homologous proteins, streptavidin and deglycosylated avidin, which have almost the same affinity for biotin as avidin does. Streptavidin has been known to be an alternative protein of avidin and has almost the same affinity for biotin. ${ }^{4}$ The structural feature of streptavidin relates to its nonglycosylated nature, while avidin contains carbohydrate chains ( $10 \%$ in weight) in the surface of the molecule. Because of the carbohydrate chains, avidin has a basic character ( $\mathrm{pI}$ of avidin; ca. 10), compared with the slightly acidic nature of streptavidin ( $\mathrm{p} I$ of streptavidin; ca.5-6). Here we report the construction of glucose oxidase (GOx) and lactate oxidase (LOx) multilayers using streptavidin on the surface of a platinum electrode. In addition, deglycosylated avidin, in which the carbohydrate chains were totally removed from the parent avidin, is also employed as a component of the GOx and LOx multilayers. The response characteristics of the multilayer-modified glucose and lactate sensors prepared using streptavidin and deglycosylated avidin are compared with those of the avidin-based enzyme biosensors.

† To whom correspondence should be addressed.

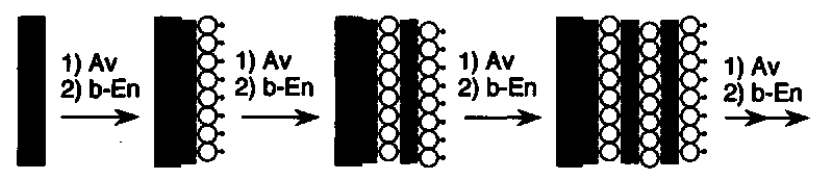

:Avidin (Av) O-Biotin-labeled enzyme (b-En)

Fig. 1 A schematic representation of a layer-by-layer deposition of avidin (or its analogues) and biotin-labeled enzyme on the electrode surface.

\section{Experimental}

Avidin (Calzyme Lab., USA), streptavidin (Southern Biotechnology Associates, Inc., USA) and deglycosylated avidin (Southern Biotechnology Associates, Inc., USA) were used as received. Glucose oxidase (GOx) (EC 1.1.3.4, Sigma Co., USA) and lactate oxidase (LOx) (EC 1.1.3.2, Boehlinger Mannheim, Germany) were labeled with several residues of biotin by the reaction with sodium sulfosuccinimidyl-6-(biotinamide)hexanoate (Vector Lab., USA), according to the reported procedure. ${ }^{1}$

A quartz-crystal microbalance (QCM) study was performed using QCA 917 system (Seiko EG \& G) equipped with a $9 \mathrm{MHz}$ Pt-coated quartz resonator (effective surface area; $0.2 \mathrm{~cm}^{2}$ ). After the resonance frequency had reached a steady-state value in the working buffer (a phosphate-buffered saline, PBS, pH 7.4) under gentle stirring, a concentrated stock solution of avidin, streptavidin, or deglycosylated avidin was added to the buffer (the final concentration; $50 \mu \mathrm{g}$ / $\mathrm{ml}$ ). The shift in the resonance frequency was recorded for 20-30 min at room temperature.

The enzyme multilayers were prepared on a Pt disk electrode ( $3 \mathrm{~mm}$ diameter), whose surface was polished thoroughly with alumina powder before use. The $\mathbf{P t}$ electrode was immersed in an avidin solution $(100 \mu \mathrm{g} / \mathrm{ml})$ for $\mathbf{2 0 ~} \mathrm{min}$ at room temperature to deposit the first avidin 
layer on the electrode surface, probably through a hydrophobic interaction ${ }^{5}$, and then was washed with PBS. The modified electrode was then immersed in a biotin-labeled GOx solution or LOx solution $(100 \mu \mathrm{g} /$ $\mathrm{ml}$ ) for $20 \mathrm{~min}$ to immobilize GOx or LOx through avidin-biotin complexation. In order to deposit more enzyme layers, this procedure was repeated using avidin, streptavidin, or deglycosylated avidin. The electrochemical response of the sensors to glucose or lactate was measured with a conventional three-electrode system at $0.6 \mathrm{~V}$ vs. $\mathrm{Ag} / \mathrm{AgCl}$ at room temperature. A $0.1 \mathrm{M}$ phosphate buffer (pH 6.8) was used for the electrochemical measurements.

\section{Results and Discussion}

It has been established that avidin is adsorbed irreversibly to the surface of $\mathrm{Pt}$ electrode to form a monomolecular layer, on which enzyme multilayers can be constructed using biotin-labeled enzymes through avidin-biotin complexation. ${ }^{1-3}$ In order to use streptavidin and deglycosylated avidin for constructing enzyme multilayers in place of avidin, it is necessary to evaluate the binding properties of these proteins to the surface of a Pt electrode. The binding properties were studied using a QCM, in which the adsorption of these proteins from solution to the surface of a Pt-coated quartz resonator was measured gravimetrically.

Figure 2 shows changes in the resonance frequency $(\Delta F)$ of QCM induced by the adsorption of streptavidin and deglycosylated avidin, together with the data for avidin. Avidin induced $\mathrm{a}-100 \pm 10 \mathrm{~Hz}$ change in the $\Delta F$, showing $550 \pm 55 \mathrm{ng} / \mathrm{cm}^{2}$ of mass increase on the quartz resonator, because the adsorption of $1 \mathrm{ng}$ substance induces a $-0.91 \mathrm{~Hz}$ change in the $9 \mathrm{MHz}$ QCM device used. ${ }^{6}$ As reported previously ${ }^{7}$, this $\Delta F$ value suggests the formation of a monomolecular layer of avidin in close packing on the Pt-coated quartz resonator, judging from the molecular weight ( $c a .68000$ ) and the molecular dimensions (ca. $4.0 \mathrm{~nm} \times 5.5 \mathrm{~nm} \times 6.0$ $\mathrm{nm}$ ) of avidn. ${ }^{4}$ On the other hand, streptavidin and deglycosylated avidin induced $-28 \pm 10$ and $-210 \pm 15 \mathrm{~Hz}$ changes in the $\Delta F$, respectively. If streptavidin forms a close-packed monomolecular layer on the quartz resonator, the $\Delta F$ should be $-85--113 \mathrm{~Hz}$ depending on the molecular orientation on the surface, calculating from the molecular weight (ca.60000) and molecular dimensions of streptavidin $(c a .4 .2 \mathrm{~nm} \times 4.2 \mathrm{~nm} \times 5.6 \mathrm{~nm}){ }^{8}$ Therefore, the QCM data mean that streptavidin is adsorbed on the Pt-coated quartz resonator to form a sub-monomolecular layer. Contrary to the formation of sub-monomolecular layer of streptavidin, deglycosylated avidin is considered to be in a multilayer (a few layers) on the surface of the quartz resonator, in view of the facts that the molecular weight of deglycosylated avidin is reported to be 62400 and that the molecular dimensions are almost the same as those of native avidin., ${ }^{9,10}$ These observations mean that streptavidin

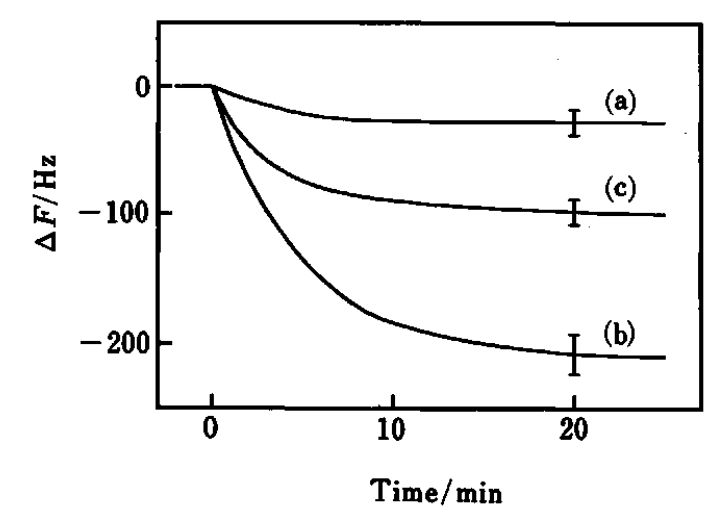

Fig. 2 Changes in resonance frequency of QCM induced by the adsorption of streptavidin (a), deglycosylated avidin (b), and avidin (c) on the surface of Pt-coated quartz resonator.

and deglycosylated avidin are not adequate to use for the first protein layer on the Pt electrode on which a layer-bylayer structure of enzyme multilayers would be further constructed.

Since the QCM study suggested the formation of a submonomolecular layer of streptavidin and a multilayer of deglycosylated avidin, in the preparation of GOx multilayer-modified enzyme sensors, the Pt disk electrode was first modified with an avidin monolayer, on which the GOx multilayers were constructed by depositing biotin-labeled GOx and streptavidin or deglycosylated avidin alternately. The GOx multilayers were assembled up to 10 layers on the Pt electrode, and their amperometric response to glucose was measured after each deposition. These biosensors exhibited an amperometric response to $1 \times 10^{-6}-3 \times 10^{-2} \mathrm{M}$ glucose with a rapid response time (10 s or faster), confirming that GOX is catalytically active in the multilayer assemblies. The most remarkable feature of these sensors is that the magnitude of the output current depended linearly on the number of the GOx layers on the $\mathrm{Pt}$ electrode. Figure 3 plots the output current of the GOx multilayer sensors to $1 \times 10^{-3} \mathrm{M}$ glucose, as a function of the deposition number of GOx. It should be noted that the magnitude of the output current of the sensors is comparable to those of the GOx/avidin multilayer-modified glucose sensor reported previously. ${ }^{1}$ For example, 10-layer GOx-modified sensors exhibited 1.0 (GOx/streptavidin) and $1.1 \mu \mathrm{A}$ (GOx/deglycosylated avidin) current to $1 \mathrm{mM}$ glucose, as compared with 1.3 $\mu \mathrm{A}$ for the GOx/avidin-modified sensor. This shows clearly that the repeated deposition of biotin-labeled GOx and streptavidin or deglycosylated avidin resulted in the multilayer assemblies of GOx, as in the case for native avidin. The linear dependence of the output current on the deposition number implies that a constant amount of GOx can be immobilized on the electrode on each deposition and that the whole GOx is catalytically active.

In a similar manner, LOx multilayer-modified lactate 


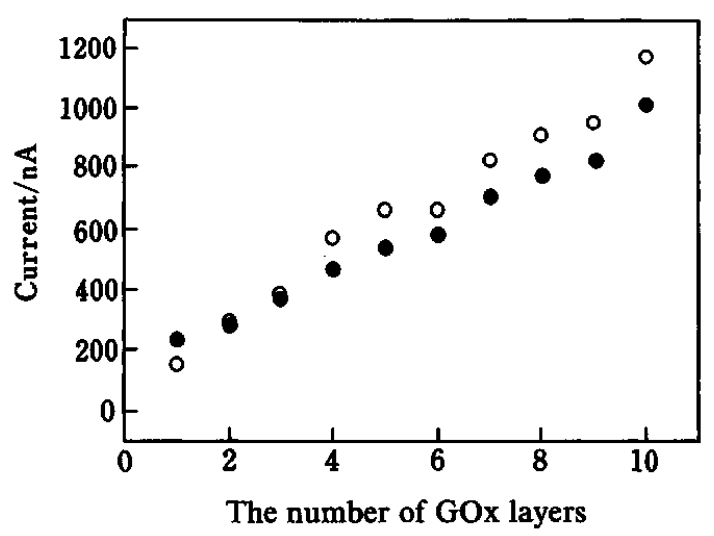

Fig. 3 The output current of the glucose sensors modified with streptavidin/GOx (O) and deglycosylated avidin $(O)$ to $1 \mathrm{mM}$ glucose as a function of the number of GOx layers.

Table 1 Effects of the number of LOx layers of the lactate sensors on the output current and the $K_{\mathrm{m}}$ app value

\begin{tabular}{ccc}
\hline Type and number of LOx layers & $i / \mu \mathrm{A}^{\mathrm{a}}$ & $K_{\mathrm{m}}{ }^{\mathrm{app}} / \mathrm{mM}$ \\
\hline LOx/streptavidin & & \\
1 & 3.4 & 0.30 \\
5 & 6.0 & 0.36 \\
10 & 9.4 & 0.43 \\
LOx/deglycosylated avidin & & \\
1 & 2.9 & 0.39 \\
5 & 7.7 & 0.40 \\
10 & 12 & 0.52 \\
1 & & \\
5 & 2.7 & 0.32 \\
10 & 7.1 & 0.40 \\
LOx/avidin & 12 & 0.54 \\
\hline
\end{tabular}

a. The output current of the sensors to $1 \mathrm{mM}$ lactate.

sensors were prepared using streptavidin and deglycosylated avidin, and their response characteristics were compared with those of the avidin-based sensors (Table 1). The output current depended nearly linearly on the number of the LOx layers for both streptavidinand deglycosylated avidin-based sensors, as well as the avidin-based sensor, although somewhat higher values than expected were observed for the monolayer-modified sensors. This shows that the catalytically-active LOx multilayers were constructed successfully using streptavidin and deglycosylated avidin. No significant difference in the magnitude of the output current was observed among the three different types of LOx multilayers. For all types of LOx multilayers, the $K_{\mathrm{m}}$ app value did not depend significantly on the number of the LOx layers, though slightly higher $K_{\mathrm{m}}$ app values were observed for the thicker multilayers.

At the present stage, we have not found any significant difference in the response characteristics and in the stability among the enzyme sensors prepared using streptavidin, deglycosylated avidin, and native avidin. It has became apparent that streptavidin and deglycosylated avidin can be used for the construction of GOx and LOx multilayer-modified biosensors.

The present work was supported in part by Grants-in-Aid (Nos. 08458282 and 09558110 ) from the Ministry of Education, Science, Sports and Culture of Japan.

\section{References}

1. T. Hoshi, J. Anzai and T. Osa, Anal. Chem., 67, 770 (1995).

2. J. Anzai, H. Takeshita, T. Hoshi and T. Osa, Chem. Pharm. Bull., 43, 520 (1995).

3. X.-Y. Du, J. Anzai, T. Osa and R. Motohashi, Electroanalysis, 8, 813 (1996).

4. N. M. Green, Adv. Protein Chem., 29, 85 (1975).

5. R. E. Ebersole, J. A. Miller, J. R. Moran, M. D. Ward, J. Am. Chem. Soc., 112, 3239 (1990).

6. H. Muramatsu, E. Tamiya, M. Suzuki and I. Karube, Anal. Chim. Acta, 217, 321 (1989).

7. J. Anzai, Y. Kobayashi, Y. Suzuki, X.-Y. Du, H. Takeshita, Q. Chen, T. Hoshi and T. Osa, submitted.

8. P. C. Weber, D. H. Ohlendorf, J. J. Wendoloski and F. R. Salemme, Science [Washington, D.C.], 243, 85 (1989).

9. R. C. Bruch and H. B. White, Biochemistry, 21, 5334 (1982).

10. H. G. Wood and R. E. Barden, Ann. Rev. Biochem., 46, 385 (1977).

(Received May 29, 1997)

(Accepted July 10, 1997) 